\title{
An Approach for Simple Linear Profile Gauge R\&R Studies
}

\author{
Liangxing Shi, ${ }^{1}$ Wei Chen, ${ }^{1}$ and Liang Fu Lu${ }^{2}$ \\ ${ }^{1}$ Department of Industrial Engineering, Tianjin University, Tianjin 300072, China \\ ${ }^{2}$ Department of Mathematics, School of Science, Tianjin University, Tianjin 300072, China
}

Correspondence should be addressed to Liang Fu Lu; liangfulv@gmail.com

Received 11 April 2014; Accepted 12 May 2014; Published 26 May 2014

Academic Editor: Xiang Li

Copyright (C) 2014 Liangxing Shi et al. This is an open access article distributed under the Creative Commons Attribution License, which permits unrestricted use, distribution, and reproduction in any medium, provided the original work is properly cited.

\begin{abstract}
Gauge repeatability and reproducibility studies are significant to quality improvement and quality control. The approaches are always applied to determine the capability of the measurement system. Much of the literature in this field mainly focuses on univariate and multivariate measurement systems. However, the state-of-the-art methods are not appropriate when the quality of a product is characterized by a profile. Therefore, this paper proposes a method for the measured values which can be characterized by a simple linear profile. In addition, the slopes and intercepts of these profiles often vary due to measurement error. Thus, the simple linear profile gauge studies can be considered as a two-response (slope and intercept) problem. $X$-values transformation is used to make the slope and intercept of each profile independent. ANOVA is utilized to estimate the variance component of measurement error and other sources of variation. Then, the criteria precision to tolerance ratio and percent R\&R are introduced to assess the simple linear profile measurement system capability. Finally, the proposed approach is applied to the spring length and elasticity measurement which demonstrates how to implement the method.
\end{abstract}

\section{Introduction}

Gauge repeatability and reproducibility (GR\&R) studies are usually adopted for determining the capability of the measurement systems. Burdick et al. [1] and Montgomery [2] proposed that one of the objectives of measurement systems capability analysis (MSCA) is to determine whether the gauge is capable. Evaluating the capability of measurement system is necessary for other researches, such as process capability analysis, control charts, and design of experiment. In practice, a reliable measurement system is significant to quality improvement and quality control.

Burdick et al. [1] reviewed the measurement system capability analysis. In their paper, the criteria, typical model, and GR\&R experiment were all introduced. Attribute data are widely applied in industry; Lyu and Chen [3] evaluated the $\mathrm{R} \& \mathrm{R}$ of a measurement system for attribute data based on the generalized linear models (GLMs). Some papers considered confidence intervals for gauge capability studies. Borror et al. [4] presented two methods to construct confidence intervals for variance components: one is the restricted maximum likelihood (REML) method and the other is the modified large sample (MLS) method. Gong et al. [5] proposed an approach by applying unweighted sums of squares method when confidence intervals for unbalanced two-factor gauge study were considered.

In practice, many products need several critical characteristics to describe their quality. For these cases, the univariate MSCA method may not be appropriate especially when the measured characteristics have some correlations. Thus, numerous studies in this field considered multivariate MSCA. An approach to analyze the two-dimensional GR\&R of imbalance measurement was introduced by Sweeney [6]. $\mathrm{He}$ concluded that the variation can be underestimated via treating such data as one-response. Majeske [7] proposed the method and the criteria for multivariate MSCA by regarding the measured values as a vector and assuming that the measured values follow a multivariate normal distribution. MANOA is applied to estimate the variance-component matrices.

Gauge repeatability and reproducibility studies are widely used in practice. Li and Al-Refaie [8] utilized the definemeasure-analyze-improve-control (DMAIC) procedure to improve the quality system involving measurements. 
Erdmann et al. [9] gave an example of GR\&R in a hospital, and the experiment is about an ear thermometer for temperature measurements.

The existing research proposed many methods for univariate and multivariate MSCA. However, the measured values in some situations can be characterized by profiles. The measurement for the elasticity and length of springs is a good example. In this case, the elasticity of spring is a simple linear profile of an independent variable: the length of spring. In this paper, an approach for the situation where the measured values can be expressed as a simple linear profile is proposed.

The remainder of this paper is organized as follows. A brief review of gauge $R \& R$ is presented in Section 2. Section 3 proposes the approach and criteria for simple linear profile gauge $R \& R$. A case study is introduced in Section 4. The last section contains our concluding remarks.

\section{The Model and Criteria for GR\&R}

Typically, gauge studies are always designed with two factors, $i$ parts and $j$ operators, and each operator measures each part $k$ times. The measured values are represented as $M_{i j k}$. These values are always treated as true values by the operators because they usually contain errors. The typical model with a two-factor design for variance analysis of $M_{i j k}$ is as follows, see Burdick et al. [1] and Montgomery [2]:

$$
M_{i j k}=\mu+p_{i}+o_{j}+(o p)_{i j}+\varepsilon_{i j k},
$$

where $i=1,2, \ldots, p, j=1,2, \ldots, o$, and $k=1,2, \ldots, r . \mu$ is the true value. $p_{i}, o_{j},(o p)_{i j}$, and $\varepsilon_{i j k}$ are all independent random variables which represent the effect of part, operator, operator-part interaction, and random error. Assume that they are all normally distributed, $p_{i} \sim N\left(0, \sigma_{p}^{2}\right), o_{j} \sim$ $N\left(0, \sigma_{o}^{2}\right),(o p)_{i j} \sim N\left(0, \sigma_{(o p)}^{2}\right)$, and $\varepsilon_{i j k} \sim N\left(0, \sigma_{e}^{2}\right)$. The variance of $M_{i j k}$ is given as follows:

$$
\begin{aligned}
\sigma_{M_{i j k}}^{2} & =\sigma_{p}^{2}+\sigma_{o}^{2}+\sigma_{(o p)}^{2}+\sigma_{e}^{2}, \\
\sigma_{G}^{2} & =\sigma_{o}^{2}+\sigma_{(o p)}^{2}+\sigma_{e}^{2},
\end{aligned}
$$

where $\sigma_{G}^{2}$ is the variance component which represents the effect of gauge.

Two criteria which are always utilized to assess the gauge capability are introduced in AIAG [10]. One is the ratio between the measurement precision estimate and the tolerance of the parts, $P / T \%$. The other is the ratio of the measurement precision estimate and the process width, $\mathrm{R} \& \mathrm{R} \%$. The criteria are formulated as follows:

$$
\begin{array}{r}
\frac{P}{T} \%=\frac{K \sigma_{G}}{\mathrm{USL}-\mathrm{LSL}} \times 100 \%, \\
\mathrm{R} \& \mathrm{R} \%=\frac{\sigma_{G}}{\sqrt{\sigma_{G}^{2}+\sigma_{p}^{2}}} \times 100 \%,
\end{array}
$$

where USL and LSL are upper specification limit and lower specification limit of the part. $K$ is either 5.15 or 6 . According to AIAG [10], the measurement system is unacceptable if

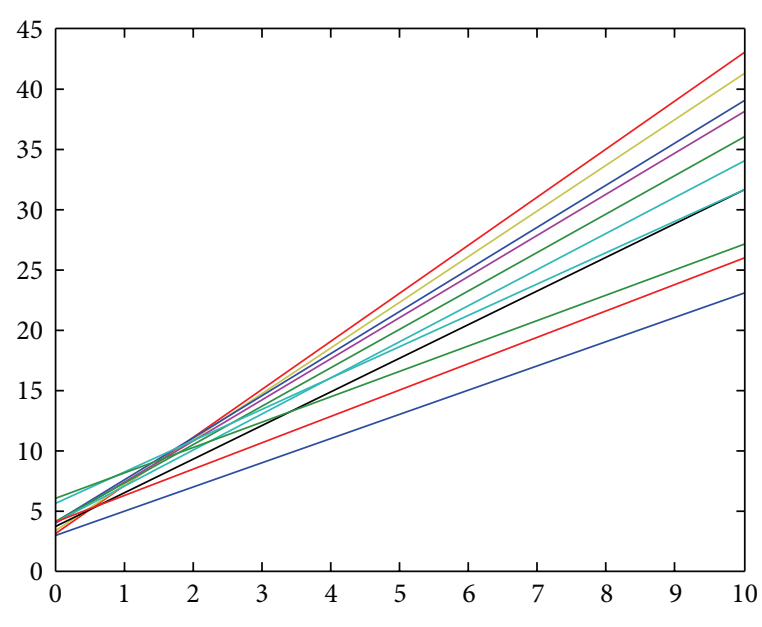

FIgURE 1: The differences of the profiles.

the value exceeds $30 \%$, and it is acceptable if the value is lower than $10 \%$. If the value is between $10 \%$ and $30 \%$, the measurement system needs to be improved. References considering these criteria include Wheeler and Lyday [11] and Woodall and Borror [12].

\section{The GR\&R Method for Simple Linear Profile}

In practice, the quality of some products can be described by simple linear profiles when they have a collection of critical characteristics to be measured. In simple linear profile gauge study, a two-factor ( $i$ parts and $j$ operators) design is considered. Each operator measures all of the parts $k$ times. The measured values are characterized by a simple linear profile:

$$
Y_{i j k}=A_{0(i j k)}+A_{1(i j k)} X_{i j k}+\varepsilon_{i j k} \text {, }
$$

where $i=1,2, \ldots, p, j=1,2, \ldots, o$, and $k=1,2, \ldots, r$. $A_{0(i j k)}$ is the intercept, and $A_{1(i j k)}$ is the slope. The random variable $\varepsilon_{i j k}$ is independent and normally distributed, $\varepsilon_{i j k} \sim$ $\left(0, \sigma^{2}\right)$. Due to measurement error, the profiles always vary, and the variation is reflected by the slopes and the intercepts. As is shown in Figure 1, where one profile represents one time measurement, the slopes and intercepts of these profiles are different.

Thus, when considering measurement error, (4) is given as follows:

$$
\begin{aligned}
Y_{i j k}= & {\left[A_{0}+p_{i A_{0}}+o_{j A_{0}}+(o p)_{i j A_{0}}+\varepsilon_{i j k A_{0}}\right] } \\
& +\left[A_{1}+p_{i A_{1}}+o_{j A_{1}}+(o p)_{i j A_{1}}+\varepsilon_{i j k A_{1}}\right] X_{i j k}+\varepsilon_{i j k},
\end{aligned}
$$

where $A_{0}$ and $A_{1}$ are constants. $p_{i A_{0}}, o_{j A_{0}},(o p)_{i j A_{0}}$, and $\varepsilon_{i j k A_{0}}$ are independent random variables which represent the effect of part, operator, operator-part interaction, and the random error on $A_{0(i j k)}$, respectively. $p_{i A_{1}}, o_{j A_{1}},(o p)_{i j A_{1}}$, and $\varepsilon_{i j k A_{1}}$ are 
independent random variables which represent the effect of part, operator, operator-part interaction, and the random error on $A_{1(i j k)}$, respectively.

3.1. The Transforming Model. For simple linear profile gauge study, $A_{0(i j k)}$ and $A_{1(i j k)}$ can be obtained easily when the measured results are profiles directly. However, it is necessary to estimate the two parameters in some cases. The leastsquare method, see Johnson and Wichern [13], is used to estimate parameters and fit profiles. The fitted profile is the following:

$$
\widehat{Y}_{i j k}=\widehat{A}_{0(i j k)}+\widehat{A}_{1(i j k)} X_{i j k} \text {, }
$$

where $\widehat{Y}_{i j k}$ is the fitted value. $\widehat{A}_{0(i j k)}$ and $\widehat{A}_{1(i j k)}$ are the leastsquare estimates of $A_{0(i j k)}$ and $A_{1(i j k)}$ which are normally distributed. $\widehat{A}_{0(i j k)}$ and $\widehat{A}_{1(i j k)}$ are correlative, and the covariance between them is $\sigma_{01}^{2}$.

Kim et al. [14] introduced a method which can make the intercept and the slope of each profile independent via transforming the $X$-values, so that the model can be simplified. An alternative form of the model in (6) is the following:

$$
Y_{i j k}=B_{0(i j k)}+B_{1(i j k)} X_{i j k}^{*}+\varepsilon_{i j k}
$$

where $X_{i j k}^{*}=X_{i j k}-\bar{X}, B_{0(i j k)}=A_{0(i j k)}+A_{1(i j k)} \bar{X}$, and $B_{1(i j k)}=A_{1(i j k)}$. In this situation, the least-squares estimator of $B_{0(i j k)}$ is $\widehat{B}_{0(i j k)}=\widehat{A}_{0(i j k)}+\widehat{A}_{1(i j k)} \bar{X}$, and the least-squares estimator of $B_{1(i j k)}$ is $\widehat{B}_{1(i j k)}=\widehat{A}_{1(i j k)}$. Both of $\widehat{B}_{0(i j k)}$ and $\widehat{B}_{1(i j k)}$ are normally distributed, and the covariance between them is zero, so $\widehat{B}_{0(i j k)}$ and $\widehat{B}_{1(i j k)}$ for each profile are independent. A separate gauge $\mathrm{R} \& \mathrm{R}$ analysis can be applied to $\widehat{B}_{0(i j k)}$ and $\widehat{B}_{1(i j k)}$, respectively.

3.2. The Model for Variance Analysis. In the alternative form, the measurement error is reflected by $\widehat{B}_{0(i j k)}$ and $\widehat{B}_{1(i j k)}$. When a two-factor design experiment is considered, the model for variance analysis of measurement error on $\widehat{B}_{0(i j k)}$ and $\widehat{B}_{1(i j k)}$ is the following:

$$
\begin{aligned}
& \widehat{B}_{0(i j k)}=\mu_{0}+p_{i B_{0}}+o_{j B_{0}}+(o p)_{i j B_{0}}+\varepsilon_{i j k B_{0}}, \\
& \widehat{B}_{1(i j k)}=\mu_{1}+p_{i B_{1}}+o_{j B_{1}}+(o p)_{i j B_{1}}+\varepsilon_{i j k B_{1}},
\end{aligned}
$$

where $\mu_{0}$ and $\mu_{1}$ are constants. $p_{i B_{0}}$ and $p_{i B_{1}}$ are independent random variables which represent the part effect on $\widehat{B}_{0(i j k)}$ and $\widehat{B}_{1(i j k)}$, respectively. $o_{j B_{0}}$ and $o_{j B_{1}}$ are independent random variables which represent the operator effect on $\widehat{B}_{0(i j k)}$ and $\widehat{B}_{1(i j k)}$, respectively. $(o p)_{i j B_{0}}$ and $(o p)_{i j B_{1}}$ are independent random variables which represent the operatorpart interaction effect on $\widehat{B}_{0(i j k)}$ and $\widehat{B}_{1(i j k)}$, respectively. $\varepsilon_{i j k B_{0}}$ and $\varepsilon_{i j k B_{1}}$ are independent random variables which represent the random error effect on $\widehat{B}_{0(i j k)}$ and $\widehat{B}_{1(i j k)}$, respectively. Assume that $p_{i B_{0}}, o_{j B_{0}},(o p)_{i j B_{0}}, \varepsilon_{i j k B_{0}}, p_{i B_{1}}, o_{j B_{1}},(o p)_{i j B_{1}}$, and $\varepsilon_{i j k B_{1}}$ are all normally distributed, where $p_{i B_{0}} \sim N\left(0, \sigma_{p 0}^{2}\right)$, $o_{j B_{0}} \sim N\left(0, \sigma_{o 0}^{2}\right),(o p)_{i j B_{0}} \sim N\left(0, \sigma_{o p 0}^{2}\right), \varepsilon_{i j k B_{0}} \sim N\left(0, \sigma_{0}^{2}\right)$, $p_{i B_{1}} \sim N\left(0, \sigma_{p 1}^{2}\right), o_{j B_{1}} \sim N\left(0, \sigma_{o 1}^{2}\right),(o p)_{i j B_{1}} \sim N\left(0, \sigma_{o p 1}^{2}\right)$, and $\varepsilon_{i j k B_{1}} \sim N\left(0, \sigma_{1}^{2}\right)$.

The variance of $\widehat{B}_{0(i j k)}$ considering the measurement error is

$$
V\left[\widehat{B}_{0(i j k)}\right]=\sigma_{p 0}^{2}+\sigma_{o 0}^{2}+\sigma_{o p 0}^{2}+\sigma_{0}^{2}
$$

The variance component of $\widehat{B}_{0(i j k)}$ for the gauge is

$$
\sigma_{G 0}^{2}=\sigma_{o 0}^{2}+\sigma_{o p 0}^{2}+\sigma_{0}^{2} \text {. }
$$
is

The variance component of $\widehat{B}_{0(i j k)}$ for the measured values

$$
\sigma_{M 0}^{2}=\sigma_{p 0}^{2}+\sigma_{G 0}^{2} .
$$
is

The variance of $\widehat{B}_{1(i j k)}$ considering the measurement error

$$
V\left[\widehat{B}_{1(i j k)}\right]=\sigma_{p 1}^{2}+\sigma_{o 1}^{2}+\sigma_{o p 1}^{2}+\sigma_{1}^{2}
$$

The variance component of $\widehat{B}_{1(i j k)}$ for the gauge is

$$
\sigma_{G 1}^{2}=\sigma_{o 1}^{2}+\sigma_{o p 1}^{2}+\sigma_{1}^{2}
$$

The variance component of $\widehat{B}_{1(i j k)}$ for the measured values is

$$
\sigma_{M 1}^{2}=\sigma_{p 1}^{2}+\sigma_{G 1}^{2}
$$

A two-factor ANOVA with an interaction term is applied to estimate these variance components.

3.3. The Assessment Criteria. Two responses $\left(\widehat{B}_{0(i j k)}\right.$ and $\left.\widehat{B}_{1(i j k)}\right)$ are obtained in the gauge study for simple linear profile. The univariate criteria $P / T \%$ and $\mathrm{R} \& \mathrm{R} \%$ can be calculated for $\widehat{B}_{0(i j k)}$ and $\widehat{B}_{1(i j k)}$, respectively. Four equations are utilized to assess the gauge capability for simple linear profile:

$$
\begin{aligned}
\frac{P}{T_{0}} \% & =\frac{K \sigma_{G 0}}{\mathrm{USL}_{0}-\mathrm{LSL}_{0}} \times 100 \%, \\
\mathrm{R} \& \mathrm{R}_{0} \% & =\frac{\sigma_{\mathrm{G} 0}}{\sqrt{\sigma_{G 0}^{2}+\sigma_{p 0}^{2}}} \times 100 \%, \\
\frac{P}{T_{1}} \% & =\frac{K \sigma_{G 1}}{\mathrm{USL}_{1}-\mathrm{LSL}_{1}} \times 100 \%, \\
\mathrm{R}_{2} \% & =\frac{\sigma_{G 1}}{\sqrt{\sigma_{G 1}^{2}+\sigma_{p 1}^{2}}} \times 100 \% .
\end{aligned}
$$

According to AIAG [10], the measurement system is unacceptable if one of the above assessed values exceeds $30 \%$. It is acceptable if the values are all lower than $10 \%$. If the values are all between $10 \%$ and $30 \%$, the measurement system needs to be improved. 
TABLE 1: Profile variance component of $\widehat{B}_{0(i j k)}$.

\begin{tabular}{lc}
\hline$\sigma_{p 0}^{2}$ & 0.007894 \\
$\sigma_{o 0}^{2}$ & 0.000009 \\
$\sigma_{o p 0}^{2}$ & 0 \\
$\sigma_{0}^{2}$ & 0.000149 \\
\hline
\end{tabular}

TABLE 2: Profile variance component of $\widehat{B}_{1(i j k)}$.

\begin{tabular}{ll}
\hline$\sigma_{p 1}^{2}$ & 0.000554 \\
$\sigma_{o 1}^{2}$ & 0.000001 \\
$\sigma_{o p 1}^{2}$ & 0.000002 \\
$\sigma_{1}^{2}$ & 0.000010 \\
\hline
\end{tabular}

\section{The Introductory Case}

An example of gauge study about spring measurement is introduced in this section. In the quality improvement, it is necessary to measure the elasticity and the length of spring. According to Hooke's law, when the spring has reached a state of equilibrium, its elasticity is a simple linear profile of the amount by which the free end of the spring is displaced from its relaxed position (when it is not stretched). In this case, three operators made three measurements on each of ten springs, $X_{i j k}$ is the length of spring after compression or elongation, and $Y_{i j k}$ is the different elasticity when the spring is of different length.

The least-square method is used to fit profile and estimate the parameters $\widehat{A}_{0(i j k)}$ and $\widehat{A}_{1(i j k)}$ after the measurement. Then, $\widehat{B}_{0(i j k)}$ and $\widehat{B}_{1(i j k)}$ in (7) can be obtained via transforming $X$-values. A two-factor ANOVA with an interaction term is utilized to estimate the variance components of $\widehat{B}_{0(i j k)}$ and $\widehat{B}_{1(i j k)}$, respectively. The variance components of $\widehat{B}_{0(i j k)}$ are shown in Table 1 . The variance components of $\widehat{B}_{1(i j k)}$ are shown in Table 2.

According to (10), (11), (13), and (14), the variance components for the gauge and the measured values of $\widehat{B}_{0(i j k)}$ and $\widehat{B}_{1(i j k)}$ are the following:

$$
\begin{gathered}
\sigma_{G 0}^{2}=0.000158, \\
\sigma_{M 0}^{2}=0.000090, \\
\sigma_{G 1}^{2}=0.000013, \\
\sigma_{M 1}^{2}=0.000567 .
\end{gathered}
$$

The upper and lower specifications on $\widehat{B}_{0(i j k)}$ are $\mathrm{USL}_{0}=$ 1.3283 and $\mathrm{LSL}_{0}=0.9717$. Then, the criteria for $\widehat{B}_{0(i j k)}$ are the following:

$$
\begin{gathered}
\frac{P}{T_{0}} \%=21.18 \%, \\
\mathrm{R} \& \mathrm{R}_{0} \%=14.03 \% .
\end{gathered}
$$

The upper and lower specifications on $\widehat{B}_{1(i j k)}$ are $\mathrm{USL}_{1}=$ -0.2464 and $\mathrm{LSL}_{1}=-0.3223$. Then, the criteria for $\widehat{B}_{1(i j k)}$ are estimated as follows:

$$
\begin{aligned}
\frac{P}{T_{1}} \% & =28.30 \%, \\
\mathrm{R} \& \mathrm{R}_{1} \% & =15.16 \% .
\end{aligned}
$$

In this case, all of the criteria are between $10 \%$ and $30 \%$. According to AIAG [10], the measurement system needs to be improved. The improvement will be made based on the gauge $\mathrm{R} \& \mathrm{R}$ experiment. In this case, the approach used to improve the capability of measurement system provides a clearer instruction. The instruction includes the precise location where the spring should be placed and the measurement operations that should be conducted. This improvement can decrease the differences among the operators.

\section{Conclusion}

Gauge repeatability and reproducibility studies are important to guarantee the validity of data, which is essential to other researches. Numerous existing studies in this area are about univariate and multivariate measurement systems, but these methods may not be suitable when the quality of a product should be characterized by a profile. This paper proposes an approach to assess the gauge capability when a simple linear profile is used to reflect product quality. Our proposed method can simplify the measurement problems effectively, especially for those with the multi-dimensional measured values. The example of spring measurement is presented in this paper which shows how to implement the proposed method. Further, more and more methods and criteria should be proposed to assess the capability of the measurement system.

\section{Appendix}

In this appendix, the original measured values of the introductory case are provided as Table 3.

\section{Conflict of Interests}

The authors declare that there is no conflict of interests regarding the publication of this paper. 
TABLE 3: The measured data of spring case.

\begin{tabular}{|c|c|c|c|c|c|c|c|}
\hline \multirow[b]{2}{*}{ Part } & \multirow[b]{2}{*}{ Operator } & \multicolumn{6}{|c|}{$X_{i j k}$} \\
\hline & & 17 & 16 & 15 & 13.5 & 12.5 & 11 \\
\hline & & \multicolumn{6}{|c|}{$Y_{i j k}$} \\
\hline 1 & 1 & 0.26 & 0.59 & 0.86 & 1.37 & 1.68 & 2.19 \\
\hline 1 & 1 & 0.24 & 0.57 & 0.86 & 1.37 & 1.66 & 2.17 \\
\hline 1 & 1 & 0.24 & 0.55 & 0.86 & 1.35 & 1.66 & 2.17 \\
\hline 1 & 2 & 0.26 & 0.57 & 0.86 & 1.35 & 1.66 & 2.17 \\
\hline 1 & 2 & 0.24 & 0.55 & 0.86 & 1.37 & 1.66 & 2.17 \\
\hline 1 & 2 & 0.26 & 0.57 & 0.86 & 1.37 & 1.66 & 2.17 \\
\hline 1 & 3 & 0.26 & 0.59 & 0.86 & 1.35 & 1.66 & 2.17 \\
\hline 1 & 3 & 0.24 & 0.55 & 0.86 & 1.37 & 1.68 & 2.17 \\
\hline 1 & 3 & 0.24 & 0.57 & 0.86 & 1.35 & 1.66 & 2.17 \\
\hline 2 & 1 & 0.46 & 0.75 & 1.06 & 1.53 & 1.84 & 2.33 \\
\hline 2 & 1 & 0.39 & 0.73 & 0.99 & 1.48 & 1.77 & 2.26 \\
\hline 2 & 1 & 0.39 & 0.73 & 0.99 & 1.48 & 1.79 & 2.24 \\
\hline 2 & 2 & 0.44 & 0.73 & 0.99 & 1.48 & 1.79 & 2.24 \\
\hline 2 & 2 & 0.44 & 0.75 & 0.99 & 1.46 & 1.79 & 2.26 \\
\hline 2 & 2 & 0.39 & 0.75 & 1.04 & 1.51 & 1.79 & 2.26 \\
\hline 2 & 3 & 0.46 & 0.75 & 1.06 & 1.48 & 1.79 & 2.26 \\
\hline 2 & 3 & 0.44 & 0.73 & 1.04 & 1.48 & 1.79 & 2.26 \\
\hline 2 & 3 & 0.39 & 0.75 & 1.02 & 1.48 & 1.79 & 2.24 \\
\hline 3 & 1 & 0.22 & 0.51 & 0.79 & 1.26 & 1.59 & 2.06 \\
\hline 3 & 1 & 0.22 & 0.48 & 0.77 & 1.24 & 1.57 & 2.04 \\
\hline 3 & 1 & 0.19 & 0.48 & 0.77 & 1.24 & 1.57 & 2.06 \\
\hline 3 & 2 & 0.22 & 0.46 & 0.75 & 1.24 & 1.55 & 2.04 \\
\hline 3 & 2 & 0.22 & 0.46 & 0.75 & 1.24 & 1.55 & 2.04 \\
\hline 3 & 2 & 0.22 & 0.46 & 0.77 & 1.24 & 1.55 & 2.06 \\
\hline 3 & 3 & 0.22 & 0.48 & 0.79 & 1.26 & 1.59 & 2.06 \\
\hline 3 & 3 & 0.22 & 0.48 & 0.77 & 1.22 & 1.55 & 2.04 \\
\hline 3 & 3 & 0.19 & 0.46 & 0.77 & 1.26 & 1.57 & 2.06 \\
\hline 4 & 1 & 0.26 & 0.55 & 0.82 & 1.24 & 1.53 & 1.97 \\
\hline 4 & 1 & 0.26 & 0.53 & 0.82 & 1.24 & 1.53 & 1.95 \\
\hline 4 & 1 & 0.26 & 0.53 & 0.79 & 1.22 & 1.53 & 1.95 \\
\hline 4 & 2 & 0.26 & 0.53 & 0.79 & 1.22 & 1.51 & 1.93 \\
\hline 4 & 2 & 0.26 & 0.53 & 0.79 & 1.22 & 1.48 & 1.88 \\
\hline 4 & 2 & 0.26 & 0.53 & 0.79 & 1.22 & 1.51 & 1.93 \\
\hline 4 & 3 & 0.26 & 0.55 & 0.82 & 1.22 & 1.48 & 1.93 \\
\hline 4 & 3 & 0.26 & 0.53 & 0.79 & 1.22 & 1.53 & 1.95 \\
\hline 4 & 3 & 0.26 & 0.53 & 0.79 & 1.22 & 1.48 & 1.93 \\
\hline 5 & 1 & 0.19 & 0.48 & 0.79 & 1.26 & 1.59 & 2.06 \\
\hline 5 & 1 & 0.19 & 0.48 & 0.79 & 1.26 & 1.55 & 2.04 \\
\hline 5 & 1 & 0.19 & 0.48 & 0.79 & 1.26 & 1.55 & 2.02 \\
\hline 5 & 2 & 0.19 & 0.51 & 0.77 & 1.24 & 1.55 & 2.04 \\
\hline 5 & 2 & 0.19 & 0.48 & 0.79 & 1.24 & 1.55 & 2.02 \\
\hline 5 & 2 & 0.19 & 0.42 & 0.73 & 1.19 & 1.48 & 2.04 \\
\hline 5 & 3 & 0.19 & 0.48 & 0.79 & 1.26 & 1.57 & 2.06 \\
\hline 5 & 3 & 0.19 & 0.46 & 0.79 & 1.24 & 1.55 & 2.02 \\
\hline 5 & 3 & 0.19 & 0.46 & 0.77 & 1.24 & 1.55 & 2.02 \\
\hline 6 & 1 & 0.28 & 0.57 & 0.82 & 1.22 & 1.46 & 1.86 \\
\hline 6 & 1 & 0.28 & 0.55 & 0.79 & 1.19 & 1.42 & 1.79 \\
\hline 6 & 1 & 0.28 & 0.55 & 0.79 & 1.19 & 1.46 & 1.84 \\
\hline 6 & 2 & 0.28 & 0.55 & 0.82 & 1.22 & 1.46 & 1.84 \\
\hline
\end{tabular}


TABLE 3: Continued.

\begin{tabular}{|c|c|c|c|c|c|c|c|}
\hline \multirow[b]{2}{*}{ Part } & \multirow[b]{2}{*}{ Operator } & \multicolumn{6}{|c|}{$X_{i j k}$} \\
\hline & & 17 & 16 & 15 & 13.5 & 12.5 & 11 \\
\hline & & \multicolumn{6}{|c|}{$Y_{i j k}$} \\
\hline 6 & 2 & 0.28 & 0.55 & 0.79 & 1.22 & 1.46 & 1.84 \\
\hline 6 & 2 & 0.28 & 0.57 & 0.82 & 1.22 & 1.46 & 1.84 \\
\hline 6 & 3 & 0.28 & 0.55 & 0.79 & 1.22 & 1.46 & 1.84 \\
\hline 6 & 3 & 0.28 & 0.55 & 0.79 & 1.19 & 1.44 & 1.82 \\
\hline 6 & 3 & 0.26 & 0.53 & 0.79 & 1.19 & 1.42 & 1.79 \\
\hline 7 & 1 & 0.19 & 0.48 & 0.77 & 1.24 & 1.53 & 1.99 \\
\hline 7 & 1 & 0.19 & 0.46 & 0.77 & 1.22 & 1.53 & 1.97 \\
\hline 7 & 1 & 0.17 & 0.46 & 0.75 & 1.22 & 1.53 & 1.97 \\
\hline 7 & 2 & 0.19 & 0.46 & 0.77 & 1.22 & 1.53 & 1.97 \\
\hline 7 & 2 & 0.17 & 0.46 & 0.77 & 1.22 & 1.53 & 1.95 \\
\hline 7 & 2 & 0.19 & 0.46 & 0.77 & 1.22 & 1.53 & 1.99 \\
\hline 7 & 3 & 0.19 & 0.46 & 0.75 & 1.22 & 1.53 & 1.97 \\
\hline 7 & 3 & 0.19 & 0.46 & 0.77 & 1.22 & 1.55 & 1.97 \\
\hline 7 & 3 & 0.17 & 0.46 & 0.75 & 1.22 & 1.51 & 1.97 \\
\hline 8 & 1 & 0.26 & 0.55 & 0.84 & 1.33 & 1.64 & 2.13 \\
\hline 8 & 1 & 0.24 & 0.53 & 0.82 & 1.31 & 1.62 & 2.08 \\
\hline 8 & 1 & 0.24 & 0.53 & 0.82 & 1.33 & 1.64 & 2.08 \\
\hline 8 & 2 & 0.26 & 0.55 & 0.82 & 1.28 & 1.62 & 2.08 \\
\hline 8 & 2 & 0.24 & 0.53 & 0.82 & 1.28 & 1.59 & 2.06 \\
\hline 8 & 2 & 0.26 & 0.57 & 0.82 & 1.33 & 1.62 & 2.08 \\
\hline 8 & 3 & 0.26 & 0.53 & 0.82 & 1.33 & 1.62 & 2.08 \\
\hline 8 & 3 & 0.26 & 0.53 & 0.82 & 1.33 & 1.64 & 2.08 \\
\hline 8 & 3 & 0.24 & 0.53 & 0.82 & 1.28 & 1.62 & 2.06 \\
\hline 9 & 1 & 0.15 & 0.44 & 0.75 & 1.22 & 1.53 & 1.99 \\
\hline 9 & 1 & 0.15 & 0.39 & 0.75 & 1.22 & 1.53 & 1.99 \\
\hline 9 & 1 & 0.13 & 0.39 & 0.73 & 1.22 & 1.53 & 1.99 \\
\hline 9 & 2 & 0.15 & 0.39 & 0.73 & 1.22 & 1.53 & 2.02 \\
\hline 9 & 2 & 0.17 & 0.42 & 0.73 & 1.22 & 1.53 & 2.02 \\
\hline 9 & 2 & 0.15 & 0.42 & 0.75 & 1.22 & 1.53 & 1.99 \\
\hline 9 & 3 & 0.15 & 0.39 & 0.73 & 1.22 & 1.53 & 2.02 \\
\hline 9 & 3 & 0.15 & 0.39 & 0.75 & 1.22 & 1.53 & 1.99 \\
\hline 9 & 3 & 0.15 & 0.39 & 0.73 & 1.19 & 1.51 & 1.97 \\
\hline 10 & 1 & 0.26 & 0.55 & 0.79 & 1.19 & 1.42 & 1.82 \\
\hline 10 & 1 & 0.26 & 0.53 & 0.79 & 1.19 & 1.42 & 1.82 \\
\hline 10 & 1 & 0.26 & 0.53 & 0.77 & 1.17 & 1.39 & 1.75 \\
\hline 10 & 2 & 0.28 & 0.55 & 0.79 & 1.19 & 1.42 & 1.82 \\
\hline 10 & 2 & 0.26 & 0.53 & 0.77 & 1.15 & 1.37 & 1.75 \\
\hline 10 & 2 & 0.28 & 0.57 & 0.79 & 1.19 & 1.44 & 1.82 \\
\hline 10 & 3 & 0.26 & 0.53 & 0.79 & 1.15 & 1.39 & 1.77 \\
\hline 10 & 3 & 0.26 & 0.53 & 0.79 & 1.15 & 1.39 & 1.75 \\
\hline 10 & 3 & 0.26 & 0.53 & 0.77 & 1.13 & 1.37 & 1.77 \\
\hline
\end{tabular}

\section{Acknowledgments}

The authors wish to thank Dr. Jinyu Yang for introducing the elementary idea of the paper. This research is supported by the National Natural Science Foundation of China (71102140) and the National Research Foundation for the Doctoral Program of Higher Education of China (20100032120030).

\section{References}

[1] R. K. Burdick, C. M. Borror, and D. C. Montgomery, "A review of methods for measurement systems capability analysis," Journal of Quality Technology, vol. 35, no. 4, pp. 342-354, 2003.

[2] D. C. Montgomery, Introduction to Statistical Quality Control, John Wiley and Sons, 2007. 
[3] J. Lyu and M.-N. Chen, "Gauge capability studies for attribute data," Quality and Reliability Engineering International, vol. 24, no. 1, pp. 71-82, 2008.

[4] C. M. Borror, D. C. Montgomery, and G. C. Runger, "Confidence intervals for variance components from gauge capability studies," Quality and Reliability Engineering International, vol. 13, no. 6, pp. 361-369, 1997.

[5] L. Gong, R. K. Burdick, and J. Quiroz, "Confidence intervals for unbalanced two-factor gauge R\&R studies," Quality and Reliability Engineering International, vol. 21, no. 8, pp. 727-741, 2005.

[6] S. Sweeney, "Analysis of two-dimensional gage repeatability and reproducibility," Quality Engineering, vol. 19, no. 1, pp. 29-37, 2007.

[7] K. D. Majeske, "Approval criteria for multivariate measurement systems," Journal of Quality Technology, vol. 40, no. 2, pp. 140$153,2008$.

[8] M.-H. C. Li and A. Al-Refaie, "Improving wooden parts' quality by adopting DMAIC procedure," Quality and Reliability Engineering International, vol. 24, no. 3, pp. 351-360, 2008.

[9] T. P. Erdmann, R. J. M. M. Does, and S. Bisgaard, "Quality quandaries: a gage R\&R study in a hospital," Quality Engineering, vol. 22, no. 1, pp. 46-53, 2010.

[10] Automotive Industry Action Group, Measurement Systems Analysis: Reference Manual, Automotive Industry Action Group, 2010.

[11] D. J. Wheeler and R. W. Lyday, Evaluating the Measurement Process, Statistical Process Controls, 1984.

[12] W. H. Woodall and C. M. Borror, "Some relationships between gage R\&R criteria," Quality and Reliability Engineering International, vol. 24, no. 1, pp. 99-106, 2008.

[13] R. A. Johnson and D. W. Wichern, Applied Multivariate Statistical Analysis, Prentice hall, Upper Saddle River, NJ, USA, 2002.

[14] K. Kim, M. A. Mahmoud, and W. H. Woodall, "On the monitoring of linear profiles," Journal of Quality Technology, vol. 35, no. 3, pp. 317-328, 2003. 


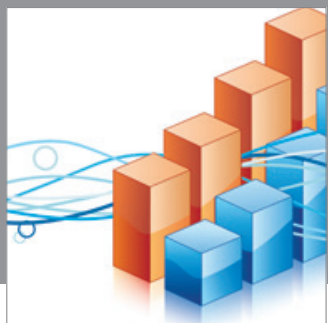

Advances in

Operations Research

mansans

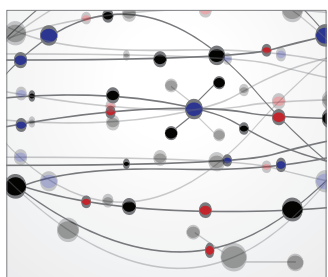

The Scientific World Journal
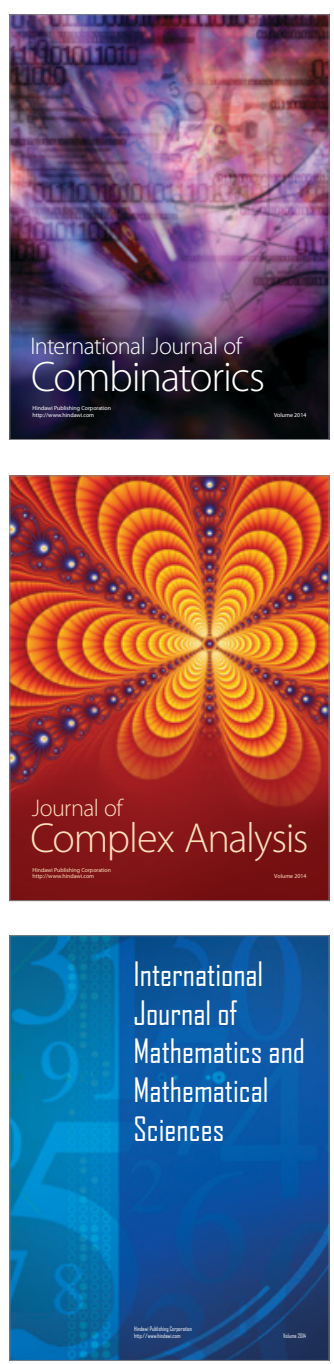
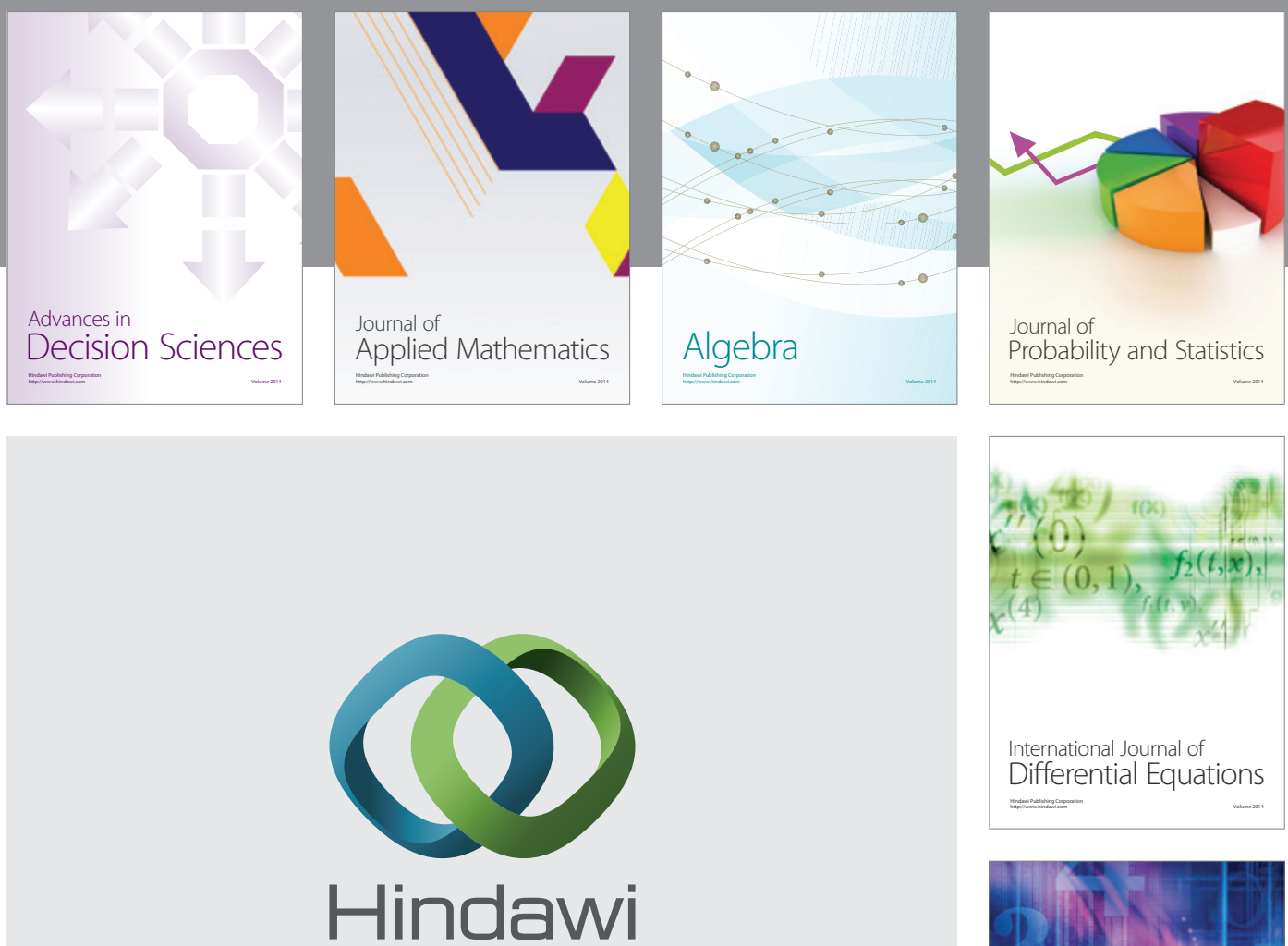

Submit your manuscripts at http://www.hindawi.com
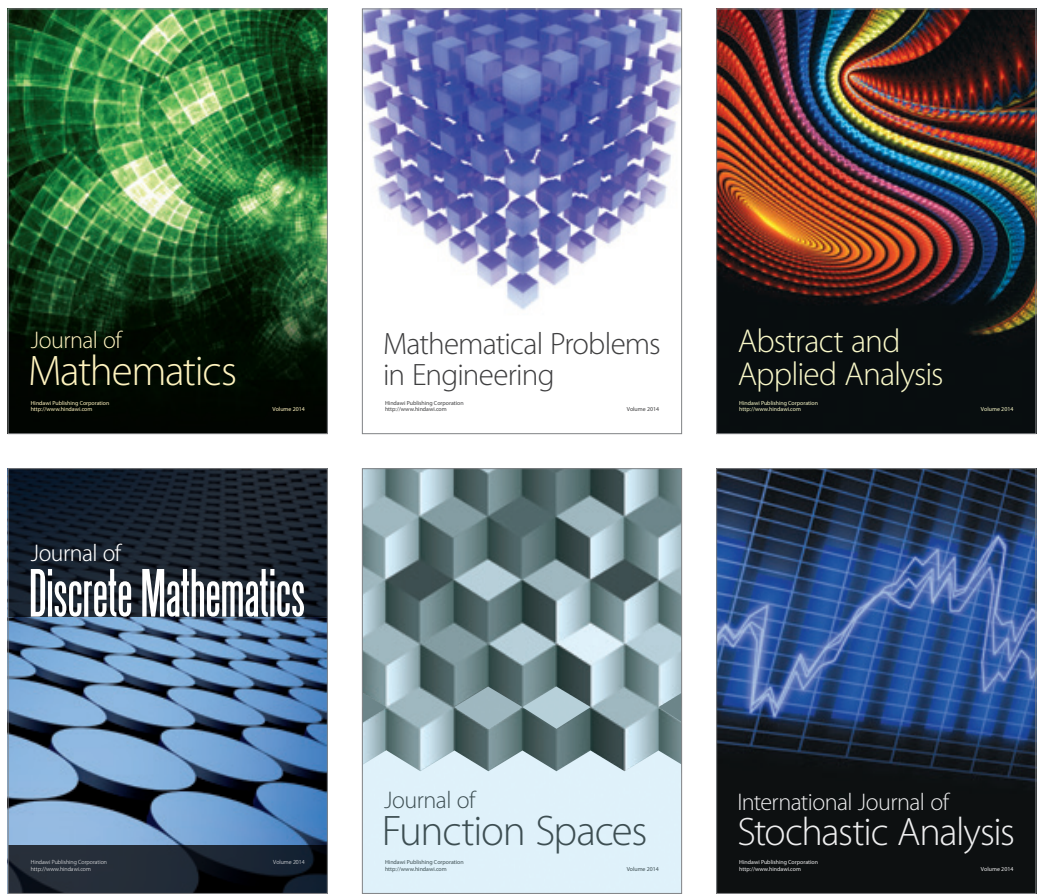

Journal of

Function Spaces

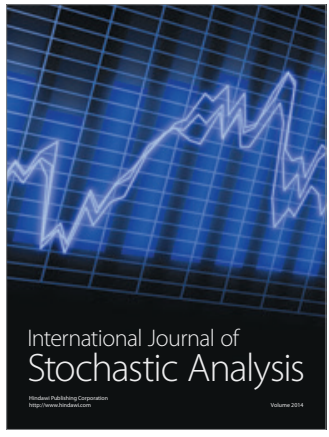

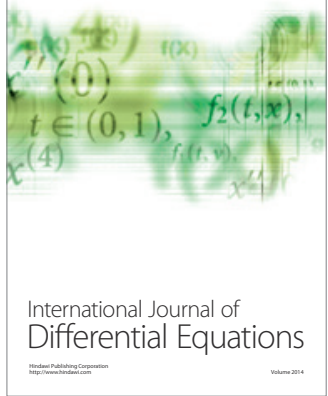
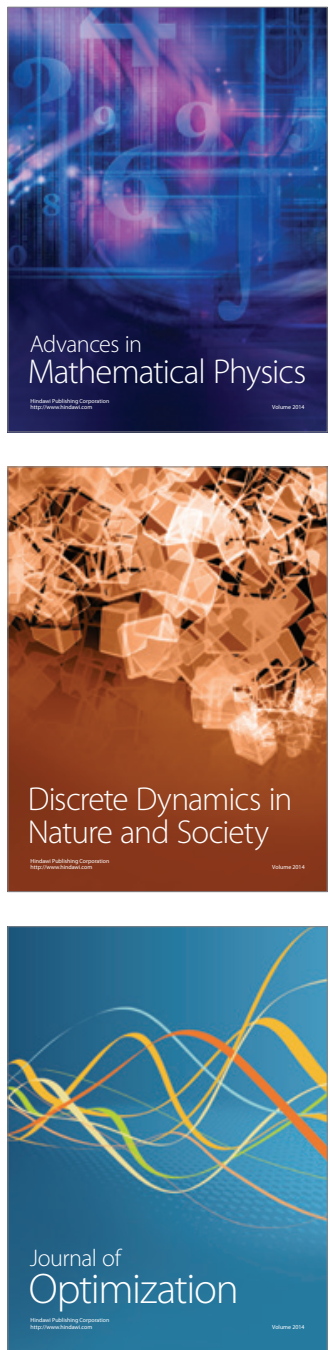